\title{
Lipoprotein(a) plasma levels are not associated with incident microvascular complications in type 2 diabetes mellitus
}

\author{
Sunny S. Singh ${ }^{1,2} \cdot$ Mardin Rashid $^{1}$ - Aloysius G. Lieverse ${ }^{2} \cdot$ Florian Kronenberg $^{3} \cdot$ Claudia Lamina $^{3}$. \\ Monique T. Mulder ${ }^{1}$ • Yolanda B. de Rijke ${ }^{4}$ • Eric J. G. Sijbrands ${ }^{1}$ • Mandy van Hoek ${ }^{1}$ (D)
}

Received: 28 August 2019 / Accepted: 11 February 2020 / Published online: 9 March 2020

(C) The Author(s) 2020

\begin{abstract}
Aims/hypothesis Microvascular disease in type 2 diabetes is a significant cause of end-stage renal disease, blindness and peripheral neuropathy. The strict control of known risk factors, e.g. lifestyle, hyperglycaemia, hypertension and dyslipidaemia, reduces the incidence of microvascular complications, but a residual risk remains. Lipoprotein (a) [Lp(a)] is a strong risk factor for macrovascular disease in the general population. We hypothesised that plasma Lp(a) levels and the $L P A$ gene SNPS rs10455872 and rs3798220 are associated with the incident development of microvascular complications in type 2 diabetes.

Methods Analyses were performed of data from the DiaGene study, a prospective study for complications of type 2 diabetes, collected in the city of Eindhoven, the Netherlands ( $n=1886$ individuals with type 2 diabetes, mean follow-up time $=6.97$ years). To assess the relationship between plasma Lp(a) levels and the LPA SNPs with each newly developed microvascular complication (retinopathy $n=223$, nephropathy $n=246$, neuropathy $n=236$ ), Cox proportional hazards models were applied and adjusted for risk factors for microvascular complications (age, sex, mean arterial pressure, non-HDL-cholesterol, HDL-cholesterol, BMI, duration of type 2 diabetes, $\mathrm{HbA}_{1 \mathrm{c}}$ and smoking).

Results No significant associations of Lp(a) plasma levels and the LPA SNPs rs10455872 and rs3798220 with prevalent or incident microvascular complications in type 2 diabetes were found. In line with previous observations the LPA SNPS rs10455872 and rs3798220 did influence the plasma Lp(a) levels.

Conclusions/interpretation Our data show no association between Lp(a) plasma levels and the LPA SNPs with known effect on $\mathrm{Lp}$ (a) plasma levels with the development of microvascular complications in type 2 diabetes. This indicates that $\mathrm{Lp}$ (a) does not play a major role in the development of microvascular complications. However, larger studies are needed to exclude minimal effects of $\mathrm{Lp}(\mathrm{a})$ on the development of microvascular complications.
\end{abstract}

Keywords Lp(a) $\cdot$ LPA $\cdot$ Microvascular complications · Nephropathy $\cdot$ Neuropathy $\cdot$ Retinopathy $\cdot$ rs $10455872 \cdot$ rs3798220 $\cdot$ SNP $\cdot$ Spline

Electronic supplementary material The online version of this article (https://doi.org/10.1007/s00125-020-05120-9) contains peer-reviewed but unedited supplementary material, which is available to authorised users.

Mandy van Hoek

m.vanhoek@erasmusmc.nl

1 Department of Internal Medicine, Erasmus MC - University Medical Center Rotterdam, Doctor Molewaterplein 40, 3015

GD Rotterdam, the Netherlands

2 Department of Internal Medicine, Maxima Medical Center, Eindhoven, the Netherlands

3 Institute of Genetic Epidemiology, Department of Genetics and Pharmacology, Medical University of Innsbruck, Innsbruck, Austria

4 Department of Clinical Chemistry, Erasmus MC - University Medical Center, Rotterdam, the Netherlands

\author{
Abbreviations \\ ApoA Apolipoprotein A \\ EAS European Atherosclerosis Society \\ Lp(a) Lipoprotein A \\ MAP Mean arterial pressure \\ MDRD Modification of Diet in Renal Disease
}

\section{Introduction}

Microvascular complications greatly reduce the quality of life of individuals with type 2 diabetes. Microvascular damage causes end-stage renal disease, blindness and peripheral neuropathy [1-3]. Strict control of risk factors (e.g. lifestyle, 


\section{Research in context}

\section{What is already known about this subject?}

- $\quad$ Lipoprotein(a) [Lp(a)] is a causal risk factor for the development of macrovascular complications in the general population, as confirmed by Mendelian randomisation studies

- New therapies have emerged to potentially decrease $L p(a)$ levels

- The risk factor profiles associated with the development of macro- and microvascular disease in type 2 diabetes are similar to each other

\section{What is the key question?}

- Is $\operatorname{Lp}(\mathrm{a})$ associated with the development of microvascular disease in type 2 diabetes?

\section{What are the new findings?}

- $\quad$ Lp(a) plasma levels were not associated with prevalent or incident microvascular endpoints

- Two LPA SNPs known to influence Lp(a) plasma levels were also not associated with microvascular endpoints

\section{How might this impact on clinical practice in the foreseeable future?}

- $\quad$ Lp(a) plays no major role in the development of microvascular complications in type 2 diabetes, so potential new therapies lowering $\mathrm{Lp}(\mathrm{a})$ may not have a large effect in lowering the burden of microvascular disease in individuals with type 2 diabetes

hyperglycaemia, hypertension and dyslipidaemia) reduces the incidence of microvascular complications. However, even when people with type 2 diabetes receive optimal treatment according to the current standards, there is a residual risk of development and progression of complications [4, 5]. Thus, there is a need to identify additional risk factors. A better understanding of these factors paves the way for better prediction, prevention and treatment. Lipoprotein(a) $[\mathrm{Lp}(\mathrm{a})]$ is a known strong risk factor for macrovascular disease in the general population [6]. New therapies have emerged that reduce $L p(a)$, and thereby could potentially decrease the burden of macrovascular disease [7]. Microvascular disease in type 2 diabetes could be a potential new target for $\mathrm{Lp}(\mathrm{a})$ lowering therapies. $\mathrm{Lp}(\mathrm{a})$ has been related to atherosclerosis and macrovascular disease [6, 8]. Microvascular diseases share underlying mechanisms with atherosclerosis and macrovascular disease $[9,10]$. We hypothesised that $\mathrm{Lp}(\mathrm{a})$ is a risk factor for microvascular disease in type 2 diabetes.

$\mathrm{Lp}(\mathrm{a})$ is an LDL-like lipoprotein with an apolipoprotein B-100 molecule, to which a unique apolipoprotein A (ApoA) is attached via a disulfide bond. $\operatorname{Lp}(\mathrm{a})$ is considered to be a more pro-thrombotic and atherogenic particle than LDL. Lp(a) plasma levels are highly heritable and are associated with the size of the ApoA protein, which is determined by the number of kringle IV type 2 (KIV) repeats [11]. Furthermore, two well investigated LPA SNPs, rs10455872 and rs3798220 influence Lp(a) plasma levels, and are associated with a lower number of Lp(a) KIV repeats [12]. In prospective, population-based studies, positive associations between Lp(a) plasma levels and macrovascular disease have been reported $[13,14]$. These findings have been confirmed using the Mendelian randomisation approach, which indicates a causal role of $\mathrm{Lp}(\mathrm{a})$. Lp(a) levels $\geq 30 \mathrm{mg} / \mathrm{dl}$ are associated with macrovascular disease. The Copenhagen City Heart Study showed a 1.6-fold increased risk for incident myocardial infarction for $\mathrm{Lp}(\mathrm{a})$ levels between 30 and $76 \mathrm{mg} / \mathrm{dl}$, corresponding to the 67th to 90th percentile, this further risk increased with higher Lp(a) levels [15]. Findings on the relationship of $\mathrm{Lp}$ (a) levels and microvascular complications in type 2 diabetes have been conflicting [16-27]. Most of these studies were of Asian populations and the generalisability of the findings to other ethnicities remains uncertain. Regarding diabetic retinopathy, two cross-sectional studies and one prospective study found that high $\mathrm{Lp}(\mathrm{a})$ levels significantly associate with diabetic retinopathy [16, 17, 23]. However, other cross-sectional studies did not find any association $[20,22]$. In diabetic nephropathy, two small prospective studies found $\mathrm{Lp}$ (a) to be positively associated with nephropathy [19, 24], whereas another prospective study by Lin et al [25] did not find any association. Similarly, the few reports for diabetic neuropathy are also conflicting $[26,27]$. The association of known LPA SNPs and ApoA isoforms with microvascular complications in type 2 diabetes has never been investigated. We therefore investigated the association of Lp(a) plasma levels and selected LPA SNPs with prevalent and incident microvascular complications of type 2 diabetes using data from the DiaGene study, a prospective case-control study on type 2 diabetes with follow-up on microvascular complications [28]. 


\section{Methods}

Study design The overall aim of the DiaGene study is to unravel the aetiology of type 2 diabetes and its complications, by identifying risk factors, e.g. genomic, glycomic and lipidomic factors. The design of the DiaGene study has been reported elsewhere [28]. Briefly, the DiaGene study is an all lines of healthcare prospective case-control study collected of individuals resident in and around the city of Eindhoven in the Netherlands. All hospitals in this area participated, as well as the centre for primary care diagnostics. Hence, virtually all individuals with type 2 diabetes in this area were approached for inclusion. Initially, 2065 patients were included by physicians at all centres. Of these, 179 patients were excluded. Reasons for exclusion were: no diabetes, type 1 diabetes, maturity-onset diabetes of the young, latent auto-immune diabetes in adults, double inclusion of the participant, post-pancreatitis diabetes, withdrawal of consent during the study period and missing informed consent. Finally, 1886 patients with type 2 diabetes and 854 controls were included in the DiaGene study, which consisted mostly of people of European descent. Written informed consent was obtained from all participants. This study was approved by the medical ethics committees of the Erasmus MC and the local hospitals in Eindhoven. For the analyses described in this paper, only data on type 2 diabetes cases were analysed.

Clinical data Laboratory data and anthropometrics were derived from medical records at inclusion. By means of a questionnaire, medical history, family history and lifestyle information were collected. Mean arterial pressure (MAP) was calculated with the formula $(2 \times$ diastolic pressure + systolic pressure)/3. Non-HDL-cholesterol was calculated with the formula non-HDL-cholesterol $=$ total cholesterol HDL-cholesterol.

Definitions of microvascular complications Diabetic retinopathy was scored by ophthalmologists as absent or present, according to the Dutch guideline for diabetic retinopathy [29]. If present, it was classified as non-proliferative, proliferative, treated with photo-coagulation or treated with intravitreal injections. Neuropathy was diagnosed by a podiatrist, neurologist or the treating physician and graded according to the Sims classification [30]. Neuropathy data were only available for those individuals with type 2 diabetes who were under surveillance in outpatient clinics $(n=796)$. Nephropathy was defined as microalbuminuria (ACR $\geq 2.5 \mathrm{mg} / \mathrm{mmol}$ for men or $\geq 3.5 \mathrm{mg} / \mathrm{mmol}$ for women) at two of three consecutive measurements, or when high microalbuminuria or macroalbuminuria was present at one measurement (ACR $\geq 12.5 \mathrm{mg} / \mathrm{mmol}$ for men and $\geq 17.5 \mathrm{mg} / \mathrm{mmol}$ for women) [28].
Lp(a) plasma concentration and Lp(a) SNPs Venous blood samples were drawn from individuals with type 2 diabetes at enrolment; after centrifugation, the plasma and buffy coat were separated. Plasma samples were directly stored at $-80^{\circ} \mathrm{C}$. $\mathrm{Lp}(\mathrm{a})$ concentrations were measured in samples that had not been defrosted previously. $\mathrm{Lp}$ (a) concentrations in plasma were measured with the KIV-2 numberindependent Randox immunoassay on a Roche Cobas c501 Chemistry Analyzer [31, 32], with an Lp(a) concentration range of 3-300 $\mathrm{mg} / \mathrm{dl}$. A total of 1850 type 2 diabetic individuals with successfully measured $\mathrm{Lp}(\mathrm{a})$ plasma levels were included in the analyses. $L P A$ SNPs rs 10455872 and rs3798220 were genotyped by using Taqman allelic discrimination assays designed and optimised by Applied Biosystems (Foster City, CA, USA). The LPA SNPs rs 10455872 and rs3798220 were successfully genotyped for $1696(90 \%)$ and 1727 (92\%) participants, respectively. Reactions were performed on the Taqman Prism 7900 HT platform.

Statistical analysis To compare baseline variables, the independent samples $t$ test was used for continuous variables with a normal distribution and the Mann-Whitney test was used for continuous variables with a non-normal distribution. Normality was assumed when skewness and kurtosis were within the range of -1 and +1 . For categorical variables, the $\chi^{2}$ test was applied. To assess the relationship between $\operatorname{Lp}(\mathrm{a})$ and microvascular endpoints, we used $\mathrm{Lp}$ (a) plasma levels as an independent primarily binary variable $(<30 \mathrm{mg} / \mathrm{dl}$ and $\geq 30 \mathrm{mg} / \mathrm{dl}$ ). This cut-off level is based on clinical studies in the general population, where an almost two times higher risk of a major adverse cardiovascular event is present when $L p(a)$ plasma levels exceed $30 \mathrm{mg} / \mathrm{dl}$ [15]. However, specifically for stroke, the 'Emerging risk factors consortium' observed a higher threshold of $L p(a)$ concentrations [14]. In addition, we assessed the relationships of the microvascular complications with $\mathrm{Lp}$ (a) as a continuous variable and in categorical quartiles. For prevalent microvascular complications at baseline, logistic regression was used to assess the relationship of $\mathrm{Lp}(\mathrm{a})$ and each of the microvascular complication endpoints. Cox proportional hazards models were used to prospectively assess the association between $\mathrm{Lp}(\mathrm{a})$ as an independent variable and each microvascular complication endpoint as a dependent variable. The prevalent cases at baseline were excluded from the prospective analyses. In addition, to assess the association of $L p(a)$ with kidney function, estimated glomerular filtration rate (eGFR) determined by the Modification of Diet in Renal Disease (MDRD) study equation [33], we performed linear regression, with \% difference in eGFR as the dependent variable and $\mathrm{Lp}(\mathrm{a})$ the independent variable. The \% difference in eGFR was calculated using the formula $\left(\left[\mathrm{eGFR}_{\text {baseline }}-\mathrm{eGFR}\right.\right.$ follow-up $\left.] / \mathrm{eGFR}_{\text {baseline }}\right) \times 100$. Model 1 was adjusted for sex and age. Model 2 was adjusted for the 
following additional risk factors for microvascular complications: MAP, non-HDL-cholesterol, HDL-cholesterol, $\mathrm{HbA}_{1 \mathrm{c}}$, BMI, duration of type 2 diabetes and smoking. In order to detect whether there was any other form of relationship we may have missed by analysing Lp(a) linearly or in quartiles, we performed non-linear spline analyses. More specifically, we applied Psplines (penalised cubic B-splines) in both the logistic regression as well as Cox models [34]. This involves selecting a high number of equidistant knots, followed by a penalty term, which is optimised via generalised cross validation to avoid overfitting. This is a data-driven and explorative approach for detecting any non-linear relationships. Models 1 and 2 described above were also applied to assess the relationship between the SNPs and each microvascular endpoint. For analysing this relationship between the SNPs rs10455872, rs3798220 and microvascular endpoints an additive genetic model was used. To assess if LPA SNPs carrier status associates with Lp(a) plasma concentration, one-way ANOVA was applied. We tested the Hardy-Weinberg equilibrium before incorporating the LPA SNPs in the analyses. A $p$ value of $<0.05$ was considered statistically significant. Statistical analyses were performed using SPSS 25.0 (IBM, Armonk, NY, USA). Non-linear spline analyses were performed using R version 3.6.0 (https:/www.r-project.org/), including packages 'mgcv' and 'survival'. For cross-sectional and prospective analyses concerning $\mathrm{Lp}(\mathrm{a})$ as binary predictor, we had $80 \%$ power to detect an OR of 1.4 and an HR of 1.5 for each microvascular endpoint, respectively. And for cross-sectional and prospective analyses with $\mathrm{Lp}$ (a) as continuous predictor, we had $80 \%$ power to detect an OR of 1.09 and an HR of 1.013 for each microvascular endpoint, respectively. Moreover, for genetic analyses, we had $80 \%$ power to detect an HR of 1.7 for each separate SNP (rs10455872 and rs3798220) separately. ESM Table 1 provides an overview of conducted post hoc power analyses [35-37].

\section{Results}

Baseline characteristics of the study population The baseline characteristics and the number (and percentage) of individuals with data missing per characteristic of the study population are shown in Table 1. The age of the participants ranged from 27 to 94 years, with a mean age of 65.2 years; $46 \%$ were female. The mean duration of type 2 diabetes before the baseline investigation was 10.02 years, mean duration of follow-up from baseline was 6.97 years. In the total population, mean Lp(a) was $27.40 \mathrm{mg} / \mathrm{dl}$ and the median was $11.00 \mathrm{mg} / \mathrm{dl}$. ESM Table 2 shows the $\mathrm{Lp}$ (a) median concentration according to each of the microvascular complications. The prevalence of retinopathy, nephropathy and neuropathy was $17 \%, 23 \%$ and $31 \%$, respectively (Table 1 ). The incidence of retinopathy, nephropathy and neuropathy was $16 \%, 19 \%$ and $49.2 \%$, respectively. Of note, data regarding the prevalence and incidence of neuropathy were available only for the 796 individuals who attended the outpatient clinic. Among the individuals free of a microvascular complication at baseline, those who developed microvascular complications during followup were significantly older $(65.0 \pm 10.4$ vs $58.6 \pm 11.6$ years, $p<0.001)$, had a later age of onset of type 2 diabetes $(54.70 \pm$ 12.61 vs $47.61 \pm 11.43$ years, $p<0.001$ ), a longer duration of type 2 diabetes $(12.44 \pm 8.89$ vs $11.05 \pm 6.28, p=0.043)$ and higher MAP $(98.41 \pm 10.14$ vs $95.54 \pm 7.85 \mathrm{mmHg}, p=$ $0.005)$ than those who were did not develop microvascular complications.

$\mathrm{Lp}(\mathrm{a})$ and prevalent complications at baseline In crosssectional analyses at baseline (ESM Table 3), individuals with type 2 diabetes with $\mathrm{Lp}$ (a) concentrations $\geq 30 \mathrm{mg} / \mathrm{dl}$ did not have significantly higher ORs than those with concentrations $<30 \mathrm{mg} / \mathrm{dl}$ for each of the microvascular endpoints in any of the models. These results did not change when the separate endpoints were taken together as one composite endpoint (OR $0.95,95 \%$ CI $0.70,1.27, p=0.71)$. Additional analyses with $\mathrm{Lp}$ (a) as a continuous variable or divided into quartiles did not change these results (ESM Tables 4, 5). Furthermore, spline analyses for Model 2 did not argue for any other non-linear relationship with prevalent microvascular endpoints (ESM Fig. 1, all $p$ values for deviation from linearity $>0.99$ ).

Lp(a) and incident complications at follow-up Table 2 shows the Cox proportional hazards models of the prospective analyses. Individuals with $\mathrm{Lp}$ (a) concentrations $\geq 30 \mathrm{mg} / \mathrm{dl}$ did not have significant HRs for any of the microvascular endpoints in any of the models. Similarly, Kaplan-Meier curves created for each microvascular endpoint did not show any significant difference between the two Lp(a) concentration groups (Fig. 1). Consideration of the three microvascular endpoints together as one composite endpoint in the Cox proportional hazards regression did not change the result (HR 1.16, 95\% CI 0.85 , $1.60, p=0.35$ ). Additional analyses with $\mathrm{Lp}(\mathrm{a})$ as a continuous variable or divided into quartiles did not change these results (ESM Tables 4 and 5). Investigation of the relationship with kidney function defined by eGFR MDRD revealed that Lp(a) was not significantly associated with percentage eGFR MDRD decline during follow-up (ESM Table 6). Furthermore, spline analyses for Model 2 also did not show any non-linear relationship of $\mathrm{Lp}(\mathrm{a})$ concentration with incident microvascular endpoints (ESM Fig. 2, all $p$ values for deviation from linearity $>0.28$ ).

LPA genotypes and $\mathrm{Lp}(\mathrm{a})$ concentrations For the entire study population independent of microvascular complications at baseline the LPA SNPs rs10455872 and rs3798220 were in Hardy-Weinberg equilibrium $\left(\chi^{2} 2.20, p>0.05\right.$ and $\chi^{2} 0.86$, $p>0.05)$, respectively. Minor allele frequencies of rs 10455872 and rs3798220 were 0.0648 and 0.0156 , 
Table 1 Baseline characteristics of the study population with successfully measured $\mathrm{Lp}(\mathrm{a})$ levels

\begin{tabular}{|c|c|c|}
\hline Characteristic & Cases $(n=1850)$ & $n(\%)$ missing data \\
\hline Female sex, $n(\%)$ & $857(46 \%)$ & $1(0.1 \%)$ \\
\hline Age (years) & $65.20(10.54)$ & $1(0.1 \%)$ \\
\hline Age of onset diabetes (years) & $54.94(11.71)$ & $118(6 \%)$ \\
\hline Duration of diabetes (years) & $10.02(8.40)$ & $118(6 \%)$ \\
\hline Duration of follow-up (years) & $6.97(2.10)$ & $11(0.6 \%)$ \\
\hline BMI, $\left(\mathrm{kg} / \mathrm{m}^{2}\right)$ & $29.50(27.00-33.05)$ & $129(7 \%)$ \\
\hline $\mathrm{HbA}_{1 \mathrm{c}}(\mathrm{mmol} / \mathrm{mol})$ & $50.82(45.46-59.57)$ & $92(5 \%)$ \\
\hline $\mathrm{HbA}_{1 \mathrm{c}}(\%)$ & $6.80(6.30-7.60)$ & $92(5 \%)$ \\
\hline MAP (mmHg) & $98.97(10.80)$ & $126(7 \%)$ \\
\hline Total cholesterol (mmol/l) & $4.29(0.93)$ & $90(5 \%)$ \\
\hline Triacylglycerols (mmol/l) & $1.43(1.02-2.04)$ & $92(5 \%)$ \\
\hline HDL-cholesterol (mmol/l) & $1.10(0.93-1.32)$ & $93(5 \%)$ \\
\hline Non-HDL-cholesterol (mmol/l) & $3.12(0.90)$ & $93(5 \%)$ \\
\hline LDL-cholesterol (mmol/l) & $2.45(0.83)$ & $120(6 \%)$ \\
\hline $\mathrm{Lp}(\mathrm{a})(\mathrm{mg} / \mathrm{dl})$ & $27.40(42.09)$ & 0 \\
\hline Lp(a) (mg/dl) & $11.00(5.00-31.55)$ & 0 \\
\hline $\mathrm{Lp}(\mathrm{a}) \geq 30 \mathrm{mg} / \mathrm{dl}, n(\%)$ & $483(26 \%)$ & 0 \\
\hline Creatinine $(\mu \mathrm{mol} / \mathrm{l})$ & $77.00(67.00-92.00)$ & $121(7 \%)$ \\
\hline eGFR (MDRD) $\left(\mathrm{ml} \mathrm{min}{ }^{-1}[1.73 \mathrm{~m}]^{-2}\right)$ & $77.36(22.48)$ & $121(7 \%)$ \\
\hline Outpatient clinic patients, $n(\%)$ & $796(43 \%)$ & - \\
\hline Smoking, $n(\%)$ & & $177(10 \%)$ \\
\hline Never & $433(26 \%)$ & \\
\hline Former & $943(56 \%)$ & \\
\hline Current & $297(18 \%)$ & \\
\hline \multicolumn{3}{|l|}{ Microvascular complications at baseline } \\
\hline Retinopathy at baseline, $n(\%)$ & $298(17 \%)$ & $105(6 \%)$ \\
\hline Nephropathy at baseline, $n(\%)$ & $375(23 \%)$ & $199(11 \%)$ \\
\hline Neuropathy at baseline, $n(\%)^{\mathrm{a}}$ & $223(31 \%)$ & $1118(60 \%)$ \\
\hline \multicolumn{3}{|c|}{ Microvascular complications during follow-up } \\
\hline Retinopathy during follow-up, $n(\%)$ & $223(16 \%)$ & $156(8 \%)$ \\
\hline Nephropathy during follow-up, $n(\%)$ & $246(19 \%)$ & $107(6 \%)$ \\
\hline Neuropathy during follow-up, $n(\%)^{\mathrm{a}}$ & $236(32 \%)$ & $1123(61 \%)$ \\
\hline
\end{tabular}

Unless stated otherwise, mean $( \pm \mathrm{SD})$ are given for normally distributed covariates. For non-normally distributed covariates, median and IQR are given

${ }^{\text {a }}$ Information only available for individuals who attended an outpatient clinic $(n=796)$ respectively (see details on genotype distribution and mean $\mathrm{Lp}$ (a) plasma concentration according to the carrier status of the LPA SNPs in Table 3). Individuals with at least one minor allele for rs10455872 and rs3798220 had significantly higher $\mathrm{Lp}$ (a) plasma concentrations compared with individuals with the wild-type genotype. The SNPs rs 10455872 and rs3798220 explained $20 \%$ and $8 \%$ of the variation in $\mathrm{Lp}$ (a) levels, respectively, and jointly explained $30 \%$ of the variance.

\section{LPA SNPs and incident microvascular complications ESM} Table 7 shows the Cox proportional hazards models for prospective analyses of the LPA SNPs, according to the additive genetic model. For both $L P A$ SNPs, carriers of the minor allele did not have significant HRs for any of the microvascular complications. These results did not change when the separate microvascular endpoints were taken together as one composite endpoint (data not shown). Furthermore, these results did not change when analysing the two SNPs together as a genetic risk score (ESM Table 8).

\section{Discussion}

In our cohort study of type 2 diabetes individuals, we did not find a cross-sectional nor a prospective association of $L p(a)$ 
Table 2 Hazard ratios for incident microvascular complications for patients with $\mathrm{Lp}(\mathrm{a})$ concentrations $\geq 30 \mathrm{mg} / \mathrm{dl}<30 \mathrm{mg} / \mathrm{dl}$ (reference group)

\begin{tabular}{|c|c|c|c|c|}
\hline & Model 1, HR (95\% CI) & $p$ value & Model 2, HR (95\% CI) & $p$ value \\
\hline \multicolumn{5}{|c|}{ Retinopathy $(n=183)$} \\
\hline$\geq 30 \mathrm{mg} / \mathrm{dl}$ & $0.94(0.69,1.26)$ & 0.66 & $1.02(0.73,1.43)$ & 0.90 \\
\hline \multicolumn{5}{|c|}{ Nephropathy $(n=185)$} \\
\hline$\geq 30 \mathrm{mg} / \mathrm{dl}$ & $1.04(0.78,1.38)$ & 0.81 & $1.03(0.73,1.44)$ & 0.88 \\
\hline \multicolumn{5}{|c|}{ Neuropathy $(n=202)$} \\
\hline$\geq 30 \mathrm{mg} / \mathrm{dl}$ & $0.99(0.74,1.33)$ & 0.95 & $0.99(0.70,1.39)$ & 0.94 \\
\hline
\end{tabular}

$n$, numbers of patients who developed the complication

Model 1: adjusted for sex and age

Model 2: additionally adjusted for MAP, non-HDL-cholesterol, HDL-cholesterol, BMI, duration of type 2 diabetes, $\mathrm{HbA}_{1 \mathrm{c}}$ and smoking (never/former/current) plasma levels and LPA SNPs rs10455872 and rs3798220 with microvascular complications.

In the general population, $\mathrm{Lp}(\mathrm{a})$ is a causal risk factor for macrovascular complications [38]. The risk factor profiles associated with the development of macro- and microvascular disease in type 2 diabetes are thought to be similar [39]. If so, microvascular disease could be an extra target for the newly developed ApoA antisense therapy [40]. In our large prospective study, elevated Lp(a) concentrations were not associated either with prevalent or incident microvascular endpoints in type 2 diabetes. Our results are strengthened by the lack of association of the LPA SNPs rs10455872 and rs3798220, which, notably, did have their known significant effects on Lp(a) plasma levels. Moreover, in our DiaGene cohort the two SNPs rs10455872 and rs3798220 explained 20\% and $8 \%$ of the variation in $\mathrm{Lp}$ (a) levels, respectively, and jointly explained $30 \%$ of the variance. This is relatively high compared with other type 2 diabetes populations (of about 1000 individuals), where rs 10455872 explained approximately $3 \%$ of the $\mathrm{Lp}(\mathrm{a})$ variation [41]. However, in the general population, Clarke et al found that these two SNPs together explained $40 \%$ of the $\mathrm{Lp}$ (a) variation [12]. Overall, in our DiaGene cohort the variance explained by the SNPs is in line with the findings in the general population. Overall, our results indicate that $\mathrm{Lp}(\mathrm{a})$-lowering therapies might not have a place in the treatment of microvascular complications in type 2 diabetes. Differences with previously reported results are unlikely to be explained by heterogeneity based on the differences between cross-sectional and prospective study designs, as we performed both and found virtually identical results. Factors that potentially contributed to the variation between the findings of the studies are ethnicity, conditions for and duration of storage of plasma samples, and method of Lp(a) measurement, which we will discuss below.

The two prospective analyses in a South Korean population by Yun et al pointed at a positive association between $\mathrm{Lp}$ (a) plasma levels and both diabetic retinopathy and nephropathy $[19,23]$. One prospective study, on kidney function decline, not strictly confined to nephropathy, in individuals of European descent with type 2 diabetes found no association with $\mathrm{Lp}$ (a) levels, in line with our findings [25]. Ethnicity is associated with differences in Lp(a) plasma levels; higher levels are found in black compared with white populations, and a significant heterogeneity has been reported across Asian populations, with lower levels observed in Chinese than in Indian populations $[42,43]$. These ethnic differences may be explained by genetic and environmental differences [44]. Notably, the ethnic differences in plasma levels of Lp(a) were not detectable at birth [45]. Macrovascular disease risk that associated with elevated Lp(a) levels also showed differential effects across different ethnicities [46]. However, it remains unknown whether and how ethnicity contributes to differential effects of $\mathrm{Lp}(\mathrm{a})$ on microvascular disease, ranging from no effect in individuals from European descent to increased risk in South Koreans.

Sample storage and method used to determine Lp(a) plasma concentration both influence measurements [32, 47]. Several studies have measured Lp(a) directly after venous blood withdrawal, whereas others first stored the samples in freezers, at different freezing temperatures. Kronenberg et al [47] showed that Lp(a) plasma levels decline over time when stored frozen, with a further decline observed with multiple thawing cycles. Samples stored at $-20^{\circ} \mathrm{C}$ declined at significantly faster rate than those stored at $-80^{\circ} \mathrm{C}$ [47]. Most of the studies so far, including the reports by Yun et al [19, 23], did not perform a KIV size-independent method, resulting in analyses with 'biased' Lp(a) levels [16-18, 20, 22, 24-27]. Marcovina et al [48] showed that these biases among methods of Lp(a) plasma measurement can substantially affect the outcomes. We have measured $\mathrm{Lp}(\mathrm{a})$ in previously unthawed samples (stored at $-80^{\circ} \mathrm{C}$ ) with a KIV size-independent method.

In our type 2 diabetes population, Lp(a) levels were relatively low compared with reports in the general population [13]. Individuals with type 2 diabetes are often in a hyperinsulinaemic state as a result of peripheral insulin resistance. Hyperinsulinaemia decreases Lp(a) plasma levels, as insulin suppresses ApoA production in hepatocytes at the post-transcriptional level [49]. One may speculate that, in type 2 diabetes, high $\operatorname{Lp}(\mathrm{a})$ levels do not contribute to 

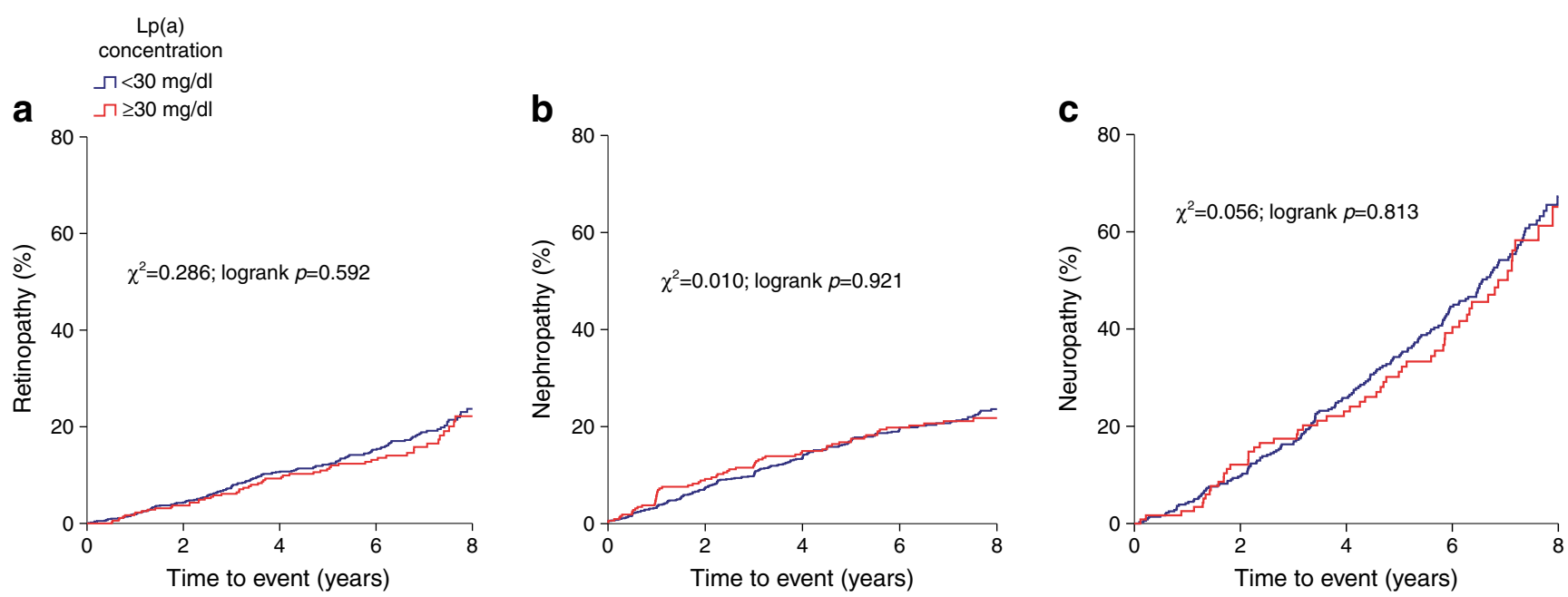

Fig. 1 Kaplan-Meier curves for the percentage of the study population who developed retinopathy (a), nephropathy (b) or neuropathy (c) during followup according to $\mathrm{Lp}(\mathrm{a})$ level

microvascular disease risk, because of other more influential competing risk factors, such as hyperglycaemia and dyslipidaemia. However, even the highest quartiles of $\mathrm{Lp}(\mathrm{a})$ levels did not display any association with microvascular complications, suggesting that there may be no role for $\mathrm{Lp}(\mathrm{a})$ in these endpoints at all.

Finally, the KIV size remains an essential property of $\mathrm{Lp}(\mathrm{a})$. The KIV size is independently inversely associated with macrovascular disease in the general population [50]. Likewise, it could be independently associated with microvascular complications in type 2 diabetes. Unfortunately, we do not have details of the KIV size. As it is known that Lp(a) plasma levels are inversely associated with the KIV size, if this effect on microvascular disease is substantial, we would have expected to find a positive association of $\mathrm{Lp}$ (a) plasma levels with microvascular complications. Also, the investigated LPA SNP rs 10455872 is strongly associated with the KIV size and showed no association with our microvascular endpoints [51]. However, we cannot exclude the possibility that the KIV size is associated with microvascular disease, and this will be the subject of ongoing studies in our population.
Strengths of our study are the prospective study design, relatively large type 2 diabetes cohort, the meticulous collection of phenotypic and risk factor data, the investigation of all microvascular endpoints and the concomitant investigation of the effects of LPA SNPs. Moreover, we used an immunoassay with the least $L p(a)$ concentration-dependent bias of the available immunoassays, and this immunoassay has a correlation coefficient $(r)$ of 0.99 with values determined by the 'gold standard' for measuring Lp(a) (an ELISA method) [32, 47]. Furthermore, we directly stored our plasma samples in minus $80^{\circ} \mathrm{C}$ and used previously unthawed samples [47]. Although we performed our study with great care, we need to consider some limitations. We had $80 \%$ power to detect an HR of 1.5 for the categorical and 1.013 for the continuous analyses. All analyses in Model 2 resulted in ORs and HRs for microvascular disease very close to 1.0 , with $95 \%$ confidence upper limits of around 1.5 for the categorical analyses and 1.005 for a continuous increase of $1 \mathrm{mg} / \mathrm{dl}$. This means that true effect sizes larger than these upper limits are highly unlikely, but effect sizes within the $95 \%$ confidence intervals cannot be excluded to become significant in much larger studies. To put this into context, a $0.8 \%$ difference
Table 3 Distribution and association LPA SNPs with $\mathrm{Lp}(\mathrm{a})$ concentrations

\begin{tabular}{lllll}
\hline SNP & $n(\%)$ & Mean Lp(a) & Median Lp(a) & $p$ value \\
\hline rs10455872 & & & & \\
$\quad$ Wild-type (AA) & $1514(87.6 \%)$ & $20.95(35.01)$ & $9.00(4.00-20.30)$ & $2.35 \times 10^{-86}$ \\
Heterozygous (AG) & $202(11.7 \%)$ & $70.58(52.21)$ & $62.00(47.00-78.50)$ & \\
Homozygous (GG) & $11(0.7 \%)$ & $158.76(62.12)$ & $167.00(96.90-203.00)$ & \\
rs3798220 & & & & \\
Wild-type (TT) & $1644(96.9 \%)$ & $25.23(37.12)$ & $11.00(5.00-30.00)$ & $4.33 \times 10^{-31}$ \\
Heterozygous (TC) & $51(3 \%)$ & $94.04(97.88)$ & $74.95(7.25-155.50)$ & \\
Homozygous $^{\mathrm{a}}$ (CC) & $1(0.1 \%)$ & $74.00(\mathrm{NA})$ & NA & \\
\hline
\end{tabular}

Mean ( \pm SD), median (IQR) are given

${ }^{\mathrm{a}} n=1, \mathrm{SD}$ not applicable (NA) 
in $\mathrm{HbA}_{1 \mathrm{c}}$, as a known continuous risk factor for microvascular disease, in the Action in Diabetes and Vascular Disease: Preterax and Diamicron MR Controlled Evaluation (ADVANCE) study resulted in an HR of 1.14 (95\% CI 1.03, 1.23) for microvascular disease after 5 years of follow-up [52]. This confidence interval does not contain the higher upper limit of the $95 \%$ CI in our study of HR 1.005. In even larger studies, our HR of 1.005 per $1 \mathrm{mg} / \mathrm{dl}$ increasing Lp(a) level could potentially become statistically significant and have an effect on microvascular disease risk, although this would be a very small effect. Furthermore, neuropathy data were only available for individuals who were seen as outpatients in the hospitals, so we cannot generalise our findings to the first line of care. In addition, data on KIV size are not available for our study population.

In conclusion, our data indicate that $\mathrm{Lp}$ (a) plasma levels do not play a major role in the development of microvascular complications in individuals from European descent with type 2 diabetes. Larger studies than ours may be able to detect very small effects of Lp(a) levels on these outcomes. Future studies taking the Lp(a) KIV size into account can further elucidate whether or not any other aspect of the $\mathrm{Lp}$ (a) particle is associated with the microvascular complications of type 2 diabetes.

Acknowledgements We want to thank Amgen Netherlands B.V. for their editorial assistance and making this research possible. Some of the data were presented as an abstract at the 87th European Atherosclerosis Society (EAS) Congress in 2019.

Data availability The datasets generated during and/or analysed during the current study are not publicly available. The raw data are subject to 'Special Categories of Personal Data (Sensitive Data)' (GDPR, Article 9), therefore raw data sharing is not in line with the privacy principles. Also, the information provided to the participants in the study states that the individual data are only accessible to the researchers, the ethical review board and (local) authorities. The informed consent given by the participants is therefore not sufficient for open access publication of indirectly identifiable data. Datasets are available from the corresponding author upon reasonable request.

Funding This research received a grant from biotechnological company Amgen Netherlands B.V. The funder provided editorial assistance only.

Authors' relationships and activities The authors declare that there are no relationships or activities that might bias, or be perceived to bias, their work.

Duality of interest The authors declare that there is no duality of interest associated with this manuscript.

Contribution statement SS completed and maintained the DiaGene study database, analysed and interpreted the data and drafted the article. MR completed the DiaGene study database and analysed and interpreted the data. AL collected data and designed the DiaGene study, initiated the research question and interpreted the data. FK interpreted the data and contributed to the spline analyses. CL interpreted the data and contributed to the spline analyses. MM supervised the laboratory measurements, interpreted the data and critically revised the manuscript. YR supervised the laboratory measurements and interpreted the data. ES collected data and designed the DiaGene study, initiated the research question and interpreted the data. MH was responsible for the coordination of the analyses, initiated the research question, collection and design of the DiaGene study, interpreted the data and drafted the manuscript. MR, AL, FK, CL, MM, YR, ES and MH critically revised the article. All authors have approved the final manuscript to be published. $\mathrm{MH}$ is the guarantor of this work and, as such, had full access to all the data in the study and takes responsibility for the integrity of the data and the accuracy of the data analysis.

Open Access This article is licensed under a Creative Commons Attribution 4.0 International License, which permits use, sharing, adaptation, distribution and reproduction in any medium or format, as long as you give appropriate credit to the original author(s) and the source, provide a link to the Creative Commons licence, and indicate if changes were made. The images or other third party material in this article are included in the article's Creative Commons licence, unless indicated otherwise in a credit line to the material. If material is not included in the article's Creative Commons licence and your intended use is not permitted by statutory regulation or exceeds the permitted use, you will need to obtain permission directly from the copyright holder. To view a copy of this licence, visit http://creativecommons.org/licenses/by/4.0/.

\section{References}

1. Bourne RR, Stevens GA, White RA et al (2013) Causes of vision loss worldwide, 1990-2010: a systematic analysis. Lancet Glob Health 1(6):e339-e349. https://doi.org/10.1016/S2214-109X(13) 70113-X

2. Nasri H, Rafieian-Kopaei M (2015) Diabetes mellitus and renal failure: Prevention and management. J Res Med Sci 20(11):11121120. https://doi.org/10.4103/1735-1995.172845

3. Bansal V, Kalita J, Misra UK (2006) Diabetic neuropathy. Postgrad Med J 82(964):95-100. https://doi.org/10.1136/pgmj.2005.036137

4. Zoungas S, Arima H, Gerstein HC et al (2017) Effects of intensive glucose control on microvascular outcomes in patients with type 2 diabetes: a meta-analysis of individual participant data from randomised controlled trials. Lancet Diabetes Endocrinol 5(6): 431-437. https://doi.org/10.1016/S2213-8587(17)30104-3

5. Holman RR, Paul SK, Bethel MA, Matthews DR, Neil HA (2008) 10-year follow-up of intensive glucose control in type 2 diabetes. $\mathrm{N}$ Engl J Med 359(15):1577-1589. https://doi.org/10.1056/ NEJMoa0806470

6. Kronenberg F, Utermann G (2013) Lipoprotein(a): resurrected by genetics. J Intern Med 273(1):6-30. https://doi.org/10.1111/j.13652796.2012.02592.x

7. Viney NJ, van Capelleveen JC, Geary RS et al (2016) Antisense oligonucleotides targeting apolipoprotein(a) in people with raised lipoprotein(a): two randomised, double-blind, placebo-controlled, dose-ranging trials. Lancet 388(10057):2239-2253. https://doi. org/10.1016/S0140-6736(16)31009-1

8. Morrisett JD (2000) The role of lipoprotein[a] in atherosclerosis. Curr Atheroscler Rep 2(3):243-250. https://doi.org/10.1007/ s11883-000-0026-Z

9. Chen CH, Henry PD (1997) Atherosclerosis as a microvascular disease: impaired angiogenesis mediated by suppressed basic fibroblast growth factor expression. Proc Assoc Am Physicians 109(4): 351-361

10. Krentz AJ, Clough G, Byrne CD (2007) Interactions between microvascular and macrovascular disease in diabetes: pathophysiology and therapeutic implications. Diabetes Obes Metab 9(6): 781-791. https://doi.org/10.1111/j.1463-1326.2007.00670.x 
11. Utermann G, Menzel HJ, Kraft HG, Duba HC, Kemmler HG, Seitz C (1987) Lp(a) glycoprotein phenotypes. Inheritance and relation to Lp(a)-lipoprotein concentrations in plasma. J Clin Invest 80(2): 458-465. https://doi.org/10.1172/JCI113093

12. Clarke R, Peden JF, Hopewell JC et al (2009) Genetic variants associated with $\mathrm{Lp}$ (a) lipoprotein level and coronary disease. $\mathrm{N}$ Eng1 J Med 361(26):2518-2528. https://doi.org/10.1056/ NEJMoa0902604

13. Kamstrup PR, Benn M, Tybjaerg-Hansen A, Nordestgaard BG (2008) Extreme lipoprotein(a) levels and risk of myocardial infarction in the general population: the Copenhagen City Heart Study. Circulation 117(2):176-184. https://doi.org/10.1161/ CIRCULATIONAHA.107.715698

14. Emerging Risk Factors C, Erqou S, Kaptoge S et al (2009) Lipoprotein(a) concentration and the risk of coronary heart disease, stroke, and nonvascular mortality. JAMA 302:412-423

15. Kamstrup PR, Tybjaerg-Hansen A, Steffensen R, Nordestgaard BG (2009) Genetically elevated lipoprotein(a) and increased risk of myocardial infarction. JAMA 301(22):2331-2339. https://doi.org/ 10.1001/jama.2009.801

16. Malaguarnera G, Gagliano C, Bucolo C et al (2013) Lipoprotein(a) serum levels in diabetic patients with retinopathy. Biomed Res Int 2013:943505

17. Tu WJ, Liu H, Liu Q, Cao JL, Guo M (2017) Association Between Serum Lipoprotein(a) and Diabetic Retinopathy in Han Chinese Patients With Type 2 Diabetes. J Clin Endocrinol Metab 102(7): 2525-2532. https://doi.org/10.1210/jc.2016-4015

18. Chopra R, Saramma JG, Mary J, Rebecca A (2007) Lipoprotein(a) as a risk factor for diabetic retinopathy in patients with type 2 diabetes mellitus. Indian J Ophthalmol 55(3):195-198. https://doi. org/10.4103/0301-4738.31939

19. Yun JS, Ahn YB, Song KH et al (2016) Lipoprotein(a) predicts a new onset of chronic kidney disease in people with type 2 diabetes mellitus. Diabet Med 33(5):639-643. https://doi.org/10.1111/dme. 12862

20. Ergun UG, Oztuzun S, Seydaoglu G (2004) Lipoprotein (A) levels in type 2 diabetic patients with diabetic retinopathy. Med J Malaysia 59(3):406-410

21. Westerhuis LW, Venekamp WJ (1996) Serum lipoprotein-a levels and glyco-metabolic control in insulin and non-insulin dependent diabetes mellitus. Clin Biochem 29(3):255-259. https://doi.org/10. 1016/0009-9120(96)00002-5

22. Mohan A, Srinivasan V, Deepa R, Mohan V (2001) Lipoprotein (a): role in diabetes and its vascular complications. J Assoc Physicians India 49:1100-1105

23. Yun JS, Lim TS, Cha SA et al (2016) Lipoprotein(a) predicts the development of diabetic retinopathy in people with type 2 diabetes mellitus. J Clin Lipidol 10(2):426-433. https://doi.org/10.1016/j. jacl.2015.12.030

24. Senba H, Furukawa S, Sakai T et al (2016) Serum lipoprotein(a) levels and diabetic nephropathy among Japanese patients with type 2 diabetes mellitus. J Diabetes Complicat 30(5):923-927. https:// doi.org/10.1016/j.jdiacomp.2016.02.006

25. Lin J, Hu FB, Mantzoros C, Curhan GC (2010) Lipid and inflammatory biomarkers and kidney function decline in type 2 diabetes. Diabetologia 53(2):263-267. https://doi.org/10.1007/s00125-0091597-z

26. Aryan Z, Afarideh M, Ghajar A, Esteghamati S, Esteghamati A, Nakhjavani M (2017) Conflicting interactions of apolipoprotein A and high density lipoprotein cholesterol with microvascular complications of type 2 diabetes. Diabetes Res Clin Pract 133:131-141. https://doi.org/10.1016/j.diabres.2017.07.037

27. Chandni R, Ramamoorthy KP (2012) Lipoprotein(a) in type 2 diabetic subjects and its relationship to diabetic microvascular complications. World J Diabetes 3(5):105-109. https://doi.org/10. 4239/wjd.v3.i5.105
28. van Herpt TTW, Lemmers RFH, van Hoek M et al (2017) Introduction of the DiaGene study: clinical characteristics, pathophysiology and determinants of vascular complications of type 2 diabetes. Diabetol Metab Syndr 9(1):47. https://doi.org/10.1186/ s13098-017-0245-x

29. Polak BC, Hartstra WW, Ringens PJ, Scholten RJ (2008) Revised guideline 'Diabetic retinopathy: screening, diagnosis and treatment' 152:2406-2413 (in Dutch)

30. Sims DS Jr, Cavanagh PR, Ulbrecht JS (1988) Risk factors in the diabetic foot. Recognition and management. Phys Ther 68(12): 1887-1902. https://doi.org/10.1093/ptj/68.12.1887

31. Marcovina SM, Koschinsky ML, Albers JJ, Skarlatos S (2003) Report of the National Heart, Lung, and Blood Institute Workshop on Lipoprotein(a) and Cardiovascular Disease: recent advances and future directions. Clin Chem 49(11):1785-1796. https://doi.org/10.1373/clinchem.2003.023689

32. Marcovina SM, Albers JJ, Scanu AM et al (2000) Use of a reference material proposed by the International Federation of Clinical Chemistry and Laboratory Medicine to evaluate analytical methods for the determination of plasma lipoprotein(a). Clin Chem 46(12): 1956-1967

33. Levey AS, Coresh J, Greene T et al (2006) Using standardized serum creatinine values in the modification of diet in renal disease study equation for estimating glomerular filtration rate. Ann Intern Med 145(4):247-254. https://doi.org/10.7326/0003-4819-145-4200608150-00004

34. Eilers PHC, Marx BD (1996) Flexible smoothing with B-splines and penalties. Stat Sci 11(2):89-102. https://doi.org/10.1214/ss/ 1038425655

35. Faul F, Erdfelder E, Buchner A, Lang AG (2009) Statistical power analyses using $G^{*}$ Power 3.1: tests for correlation and regression analyses. Behav Res Methods 41(4):1149-1160. https://doi.org/ 10.3758/BRM.41.4.1149

36. Sham PC, Purcell SM (2014) Statistical power and significance testing in large-scale genetic studies. Nat Rev Genet 15(5):335346. https://doi.org/10.1038/nrg3706

37. Pounds S (2016) Package "FDRsampsize". Available from https:// cran.r-project.org/web/packages/FDRsampsize/FDRsampsize.pdf

38. Kronenberg F (2016) Human genetics and the causal Role of lipoprotein(a) for various diseases. cardiovasc Drugs Ther 30(1): 87-100. https://doi.org/10.1007/s10557-016-6648-3

39. Wandell PE (1999) Risk factors for microvascular and macrovascular complications in men and women with type 2 diabetes. Scand J Prim Health Care 17(2):116-121. https://doi. org/10.1080/028134399750002755

40. Schreml J, Gouni-Berthold I (2017) Apolipoprotein(a) Antisense oligonucleotides: a new treatment option for lowering elevated lipoprotein(a)? Curr Pharm Des 23(10):1562-1570. https://doi. org/10.2174/1381612823666170125160108

41. Qi Q, Workalemahu T, Zhang C, Hu FB, Qi L (2012) Genetic variants, plasma lipoprotein(a) levels, and risk of cardiovascular morbidity and mortality among two prospective cohorts of type 2 diabetes. Eur Heart J 33(3):325-334. https://doi.org/10.1093/ eurheartj/ehr350

42. Virani SS, Brautbar A, Davis BC et al (2012) Associations between lipoprotein(a) levels and cardiovascular outcomes in black and white subjects: the Atherosclerosis Risk in Communities (ARIC) Study. Circulation 125(2):241-249. https://doi.org/10.1161/ CIRCULATIONAHA.111.045120

43. Banerjee D, Wong EC, Shin J, Fortmann SP, Palaniappan L (2011) Racial and ethnic variation in lipoprotein (a) levels among Asian Indian and Chinese Patients. J Lipids 2011:291954

44. Enkhmaa B, Anuurad E, Berglund L (2016) Lipoprotein (a): impact by ethnicity and environmental and medical conditions. J Lipid Res 57(7):1111-1125. https://doi.org/10.1194/jlr.R051904 
45. Rifai N, Heiss G, Doetsch K (1992) Lipoprotein(a) at birth, in blacks and whites. Atherosclerosis 92(2-3):123-129. https://doi. org/10.1016/0021-9150(92)90271-h

46. Guan W, Cao J, Steffen BT et al (2015) Race is a key variable in assigning lipoprotein(a) cutoff values for coronary heart disease risk assessment: the Multi-Ethnic Study of Atherosclerosis. Arterioscler Thromb Vasc Biol 35(4):996-1001. https://doi.org/10.1161/ ATVBAHA.114.304785

47. Kronenberg F, Lobentanz EM, Konig P, Utermann G, Dieplinger H (1994) Effect of sample storage on the measurement of lipoprotein[a], apolipoproteins B and A-IV, total and high density lipoprotein cholesterol and triglycerides. J Lipid Res 35(7):13181328

48. Marcovina SM, Albers JJ (2016) Lipoprotein (a) measurements for clinical application. J Lipid Res 57(4):526-537. https://doi.org/10. 1194/jlr.R061648

49. Rainwater DL, Haffner SM (1998) Insulin and 2-hour glucose levels are inversely related to $\mathrm{Lp}$ (a) concentrations controlled for
LPA genotype. Arterioscler Thromb Vasc Biol 18(8):1335-1341. https://doi.org/10.1161/01.ATV.18.8.1335

50. Sandholzer C, Saha N, Kark JD et al (1992) Apo(a) isoforms predict risk for coronary heart disease. A study in six populations. Arterioscler Thromb 12(10):1214-1226. https://doi.org/10.1161/ 01.atv.12.10.1214

51. Schmidt K, Noureen A, Kronenberg F, Utermann G (2016) Structure, function, and genetics of lipoprotein (a). J Lipid Res 57(8):1339-1359. https://doi.org/10.1194/jlr.R067314

52. Heller SR, ADVANCE Collaborative Group (2009) A summary of the ADVANCE Trial. Diabetes Care 32(Suppl 2):S357-S361. https://doi.org/10.2337/dc09-S339

Publisher's note Springer Nature remains neutral with regard to jurisdictional claims in published maps and institutional affiliations. 